Research Article

\title{
Optimization of Brand Marketing Strategy of Intelligent Technology under the Background of Artificial Intelligence
}

\author{
Yishu Liu ${ }^{1}$ and Weixiong Chen $\mathbb{D}^{2}$ \\ ${ }^{1}$ School of Economics and Management, Nanchang Normal College of Applied Technology, Nanchang 330108, Jiangxi, China \\ ${ }^{2}$ School of Economics, Fujian Normal University, Fuzhou 350007, Fujian, China \\ Correspondence should be addressed to Weixiong Chen; chenwx512@fjnu.edu.cn
}

Received 3 August 2021; Revised 26 August 2021; Accepted 1 September 2021; Published 14 September 2021

Academic Editor: Sang-Bing Tsai

Copyright (c) 2021 Yishu Liu and Weixiong Chen. This is an open access article distributed under the Creative Commons Attribution License, which permits unrestricted use, distribution, and reproduction in any medium, provided the original work is properly cited.

\begin{abstract}
With the progress of Internet technology and the rapid development of artificial intelligence, intelligent technology products rapidly grow up. However, influenced by many factors, the marketing status of this kind of products in recent years is not ideal, for example, big data leakage and other issues. Especially in recent years, because of the greater market competition, the marketing of intelligent technology brands is facing greater problems. The purpose of this study is to analyze the current situation of brand marketing of intelligent technology under the background of artificial intelligence and propose the optimization strategy of brand marketing of such products, so as to promote the continuous improvement of brand marketing effect of intelligent technology. Based on artificial intelligence and the concept of brand marketing, through intelligent technology brand marketing investigation and experiment, the intelligent technology brand marketing status and current problems are analyzed; from marketing, product type, and product price, three angle analysis of the factors that influence the marketing and Internet marketing, product quality, product pricing, and product development is put forward from the four angles of specific strategy of optimization of smart technology products' brand marketing. The experimental results show that the year-on-year growth rate of smart technology brand turnover is decreasing at the present stage. The increase rate of the turnover in 2019 is only 3.24\%, far lower than that in 2015 , and the growth rate of the turnover decreases by about 33\%. At present, there are many problems in the marketing strategy of intelligent technology brands, and the optimization of marketing strategy is an urgent problem to be solved.
\end{abstract}

\section{Introduction}

Because of the continuous improvement of deep learning and computer hardware system, artificial intelligence is gradually developing towards a new stage. Artificial intelligence covers a wide range of technologies, mainly the study of computer thinking and intelligence, to promote that the computer can gradually simulate some of the human behaviors. Image recognition, robotics, and language processing are all research directions in this field. In recent years, because of the continuous development of artificial intelligence applications, the commercialization trend of artificial intelligence has become more and more obvious. Based on this background, various intelligent technology brands have been rising in the market. In the face of fierce market competition, the sales of intelligent technology brands have become a common concern of the society. The rapid progress of artificial intelligence has not only promoted technological innovation and changed people's daily life but also promoted the transformation of business model. For example, face recognition technology is applied to beverage vending machines. In the field of science and technology, in addition to specific technologies, marketing strategies also play an important role in the development and survival of enterprises. Technology plays a decisive role in product quality, while marketing strategies play a decisive role in product market value.

Based on the background of artificial intelligence and market commercialization, the rise of any intelligent 
technology brand is closely related to brand marketing [1]. With the continuous development of intelligent technology, the competition of intelligent technology products becomes increasingly fierce and the sales of such products also face certain problems. The marketing problem based on this intelligent technology brand has become an urgent problem to be solved [2, 3]. Based on this, scholars at home and abroad have carried out a series of research studies and achieved relevant research results [4]. In the literature [5], millet intelligent household products as the research object under the background of artificial intelligence millet smart marketing strategy of science and technology are analyzed and the product quality, expand overseas market, and the ecological development put forward the marketing strategy of three angle optimization suggestions, which provide a certain reference value to other intelligent technology brand marketing. In the literature [6], based on the background of the development of deep learning technology, the authors analyze the impact of the weak sales growth of intelligent technology products and expound the factors affecting the marketing effect from the perspectives of sales mode and product competitiveness. In the literature [7], the authors start from the background of the rapid development of the Internet and put forward suggestions on the realization of effective brand marketing of intelligent technology based on the concept of mobile marketing Internet.

For the realization of the smart brand marketing strategy of science and technology under the background of artificial intelligence optimization and to promote the continuous increase of its marketing effect, based on the concept of artificial intelligence and brand marketing, through intelligent technology brand marketing investigation and experiment, the intelligent technology brand marketing present situation and current problems are analyzed; from marketing, product type, and product price, three angle analysis of the factors that influence the marketing and the Internet marketing, product quality, product pricing, and product development is put forward from the four angles of optimizing concrete strategy of intelligent technology products brand marketing $[8,9]$. The research in this paper, on the one hand, is conducive to the improvement of marketing effect and, on the other hand, lays a certain guiding foundation for the subsequent research in related aspects [10].

\section{Method}

2.1. Artificial Intelligence. Artificial intelligence, referred to as AI, is an emerging discipline developed based on Internet technology [11]. In essence, artificial intelligence is an important part of computer science, which aims to explore the root of intelligence and produce intelligent machines similar to human intelligence in this lifetime [12]. The research fields of AI mainly include language image recognition, expert system, robot development, and natural language processing. Artificial intelligence is developed on the basis of the rapid development of information technology $[13,14]$. It is an interdisciplinary subject established by a variety of disciplines, including computer science, information theory, psychology, and neurophysiology [15]. The intelligent behaviors that intelligent robots can perform are usually called artificial intelligence $[16,17]$. The activities related to human intelligence include a series of thinking activities such as recognition, judgment, reasoning, and thinking. In the development of contemporary science and technology, artificial intelligence has shown great advantages, which are mainly reflected in the following aspects [18]: first, artificial intelligence has realized the supplement of human intelligence. Based on the progress of science and technology, human beings have created many complex machines, which have expanded the functions of their hands and feet [19-21]. Based on the corresponding extension of human thinking organs and intellectual functions, artificial intelligence has emerged as times require [22]. Therefore, artificial intelligence belongs to the inevitable result of machine evolution and is the materialized reflection of human intelligence in science and technology, which can continuously deepen human's understanding of the world. Second, artificial intelligence promotes the rapid liberation of human intelligence. The development of artificial intelligence represents the development of a new round of industrial revolution and plays an important role in the improvement of all aspects of human social life.

The purpose of artificial intelligence research is to equip computers with human intelligence, so that they have certain abilities of autonomic computing, learning, and thinking and can replace human beings to complete some complex and difficult work [23]. Artificial intelligence mainly includes computer vision, natural language processing, speech processing, intelligent robot, and other related research fields. The current artificial intelligence system has been gradually established and applied to various fields [24, 25]. The application of artificial intelligence in brand marketing is based on algorithms such as PCA, LBP, migration learning, and measurement learning to achieve the application of graphics recognition, speech recognition, big data, and other technologies [26]. AI is an auxiliary tool for brand marketing. It can help marketers evaluate the marketing effect and make the marketing strategy more targeted.

\subsection{Research Algorithms and Methods}

2.2.1. Intelligent Algorithm. Suppose the number of features extracted from the analysis data of artificial intelligence physical education application is $M$, and they constitute an original feature set $F=\{F 1, F 2, \ldots, F M\}$, where $m$ represents the number of original features. Since some features have little effect on the analysis result, they can be removed. If a feature is selected, its value will be 1 ; otherwise, its value will be $0 . \mathrm{sm}\}$, si value is 1 or 0 . The mathematical model of artificial intelligence in the application performance analysis feature selection of intelligent technology products is as follows:

$$
S=\{s 1, s 2, \ldots . . s n\}, s i \in\{0,1\}, \quad i=1,2, \ldots ., m .
$$

In this paper, support vector machine is selected as the application performance analysis algorithm of artificial 
intelligence in the field of intelligent science and technology. The decision-making method of artificial intelligence application performance analysis in the field of intelligent science and technology is as follows:

$$
f(x)=\sum_{i=1}^{n} \alpha i \gamma i k(x i, x)+b .
$$

Among them, threshold $b$ and artificial intelligence directly affect the effect of application performance analysis in the field of intelligent technology, which needs to be optimized. From equations (1) and (2), it can be seen that the purpose of feature selection optimization and algorithm parameter optimization is to realize the application performance of high-precision artificial intelligence in the field of intelligent science and technology, and its characteristics are related to the parameters of support vector machine.

2.2.2. SWOT Analysis. SWOT analysis is mainly used to analyze the four major environmental factors of an enterprise, including disadvantages, advantages, opportunities, and challenges. As a common way to analyze the organizational environment, SWOT analysis is widely used in the strategic development of an enterprise. This method mainly analyzes the internal and external competition conditions of the organization. Corporate culture, organization, and products are all internal factors, and the industry environment, market conditions, and competition environment are all external environments. By enumerating and classifying the internal and external environments in detail and forming a matrix according to a certain way, the internal and external environments of an enterprise can be accurately analyzed and specific strategic planning can be made. In this paper, with the help of SWOT analysis, the author makes a comprehensive analysis on the marketing situation of intelligent technology products at the present stage and marketing factors and comes to the conclusion that the problem of weak sales at the present stage is the biggest problem to be overcome in the development process of intelligent technology products at the present stage and the sales amount of intelligent technology is far from shrinking in the proportion of market share. At the same time, this paper analyzes the factors affecting the insufficient product sales and puts forward the intelligent technology brand marketing strategy based on this.

\subsection{Brand Marketing Strategy}

2.3.1. Brand Building Method. The establishment of enterprise brand is not accomplished in an action, and it needs to follow certain steps. The steps of brand establishment mainly include the following aspects: first, analyze the industry environment and explore the concept of separation. Starting from the similar competition existing in the market, the position of competitors in the hearts of consumers is clarified, and the advantages and disadvantages of competitors are accurately found. Identify a particular concept to differentiate itself from its competitors. Second, ensure product quality. Product quality is the basis of establishing a good image. The quality concept proposed in this paper belongs to the comprehensive quality concept, including engineering quality and cultural quality. Third, ensure the integration and continuity of communication and application. If we want to implant the brand into the hearts of consumers, we must strive to spread the brand concept.

2.3.2. Implementation of Brand Marketing. The concept of brand marketing is to deepen consumers' understanding of enterprise brands and related products, so as to improve the competitiveness of enterprises. The construction of a huge three-dimensional sales network is not a high-level form of marketing. High-level marketing is to build an invisible marketing network in the hearts of consumers with the help of the symbolic role of the brand, so as to realize the spiritual transmission between the product and consumers and improve the recognition degree of consumers and investors for the product. Brand marketing refers to the process in which an enterprise creates brand value based on the customer's demand for the brand, thus generating brand benefits. Marketing means show the good image, reputation, and popularity of the enterprise in front of consumers, so as to establish solid brand awareness in the hearts of consumers. High level of product quality is the foundation of brand marketing. Only when the quality reaches the standard can the brand be quickly recognized by consumers. The foundation of brand establishment includes two aspects, namely, tangible products and intangible services. Specific products refer to the design, packaging, name, and other contents related to the product entity "Intangible Service," which refers to the service provided by the seller to the buyer to meet the needs of consumers after the products are sold. At the present stage, the overall quality of intelligent technology products is not much worse. From the perspective of consumers, consumers usually pay more attention to the service aspect of merchants. Therefore, from the perspective of long-term competition, enterprises must implement brand marketing in order to achieve long-term good development. From the perspective of the enterprise, the marketing method of the enterprise must take into account the interests of the enterprise and customer satisfaction.

Brand is the symbol of enterprise image and the symbol of enterprise itself, product quality, and service. In essence, brand is not only a good embodiment of enterprise comprehensive strength but also an intangible asset of enterprise management level. It plays an important role in enterprise development and competition. Only successful construction and use of the brand can occupy a favorable position in the market. Especially since the economic globalization, some brands have been rising rapidly, and the competition between products has gradually shifted to the competition between enterprises. Therefore, the implementation of effective brand marketing is the top priority of enterprise development at this stage. In essence, a brand is a symbol, which is the concentration of key information of various types of enterprises, realizing the integration of credibility, 
products, technology, culture, and other information. In the society, it is necessary to create a good reputation and image of the enterprise, so that it is recognized by the public, in the hearts of consumers to depict the enterprise symbol is the process of brand building. One cannot simply use investment as a measure of brand value. At the present stage, the core competition of enterprises is gradually transferred to the brand competition, which is the decisive factor of the competitiveness of enterprises.

\section{Intelligent Technology Brand Marketing Survey}

In order to optimize the brand marketing strategy of intelligent technology, the author conducted an investigation and analysis experiment on intelligent technology brands, which mainly consist of two parts: the first part is an investigation on the market situation of intelligent technology products. The author consulted the economic yearbook and relevant economic data websites and collected and sorted out the development data and market share data of China's intelligent technology products from 2015 to 2019. The second part includes smart technology brand marketing data collection. First, conduct field research on intelligent technology product enterprises, including sales data, marketing methods, and marketing effects of intelligent technology products, and finally form a research report. Second, using the method of online questionnaire, this paper analyzes the current situation and existing problems of intelligent technology product marketing. The subjects of the questionnaire include marketers, experts, and consumers. The questionnaire includes marketing methods, marketing factors, and marketing problems. In this study, 300 online questionnaires were distributed and 279 valid questionnaires were recovered. After the completion of the survey and analysis experiment, the SPSS statistical analysis software was used to analyze and sort out the collected data, and the computer graphics software was used to draw the data chart. Based on the analysis of the data chart, the relevant conclusions of this paper were drawn. In addition, in order to ensure the accuracy and scientificity of the research results, the author also consulted WanFang, CNKI, and other data websites, collected 12 relevant research literatures, and sorted out the data in these literatures. These data provide sufficient data support for the experimental research of this paper.

\section{Intelligent Technology Brand Marketing}

\subsection{Marketing Status Analysis}

4.1.1. Market Status of Intelligent Technology Products. Figure 1 shows the development speed of China's intelligent technology products and the market share of such products from 2015 to 2019. From the data in the figure, it can be seen that, from 2015 to 2019, the development speed of intelligent technology has been continuously improved, gradually increasing from $41.32 \%$ in 2015 to $87.13 \%$. Because of the rapid development of artificial intelligence technology, the

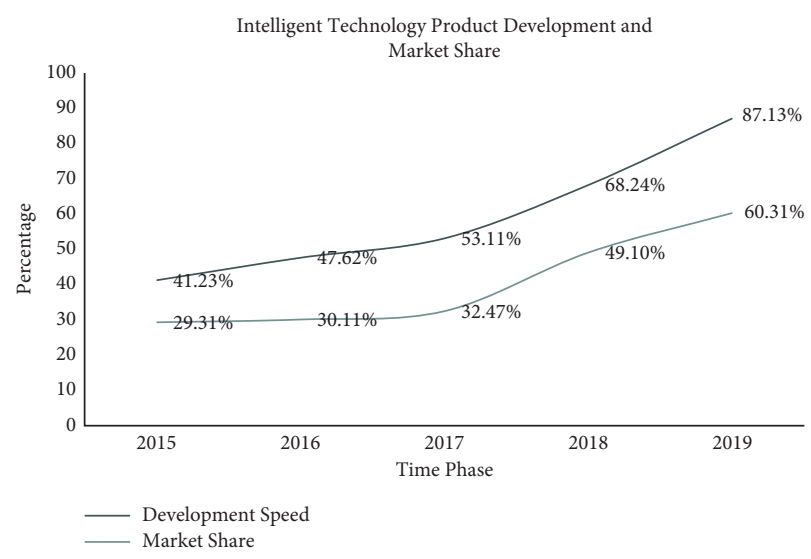

FIGURE 1: Development and market share of intelligent technology products from 2015 to 2019.

development speed of intelligent technology products has been greatly improved in recent two years. It will increase by about $15 \%$ and $19 \%$ in 2018 and 2019, respectively. In addition, from 2015 to 2019 , the share of intelligent technology products in the life cycle of Chinese science and technology products gradually increased, from $29.13 \%$ in 2015 to $60.31 \%$ in 2019, especially the market share of such products increased rapidly in the past two years. The market demand of this surface intelligent technology product is big, and the recognition degree of consumer is relatively high.

\subsubsection{Intelligent Technology Brand Marketing Data.} Table 1 and Figure 2 both show the year-on-year growth of the turnover of smart technology brands from 2015 to 2019. It can be seen from the data in the chart that the year-on-year growth rate of the turnover of smart technology brands is decreasing from 2015 to 2019 . The increase rate of the turnover in 2019 is only $3.24 \%$, which is much lower than that in 2015, and the growth rate of the turnover decreases by about $33 \%$. In addition, it can be seen from the line chart in Figure 2 that the growth rate of smart technology brands' turnover basically shows a straight downward trend. In addition, we can also see from the data in the figure that, in recent years, the share of the sales of smart technology in the market has been declining, gradually dropping from $47.21 \%$ to $21.05 \%$. To sum up, the problem of weak sales growth is the key problem that smart technology brand marketing is facing at the present stage, and it belongs to the problem that needs to be solved urgently.

4.1.3. Analysis of Marketing Influencing Factors. Figure 3 shows the statistics of influencing factors of intelligent technology marketing. It can be seen from the data in the figure that the factors affecting the marketing effect of intelligent technology at the present stage mainly include three aspects: marketing method, product type, and product price. The author will make a detailed analysis of these three factors influencing the marketing effect. First, the marketing method is backward. The backwardness of marketing is mainly reflected in the following aspects. The first is the 
TABLE 1: Growth status of smart technology brand turnover.

\begin{tabular}{lrrrrr}
\hline Year & 2015 & 2016 & 2017 & 2018 & 2019 \\
\hline $\begin{array}{l}\text { Year-on-year growth of } \\
\text { turnover (\%) }\end{array}$ & 36.28 & 32.14 & 20.01 & 10.31 & 3.24 \\
*Data are based on the results of an experimental investigation \\
\hline
\end{tabular}

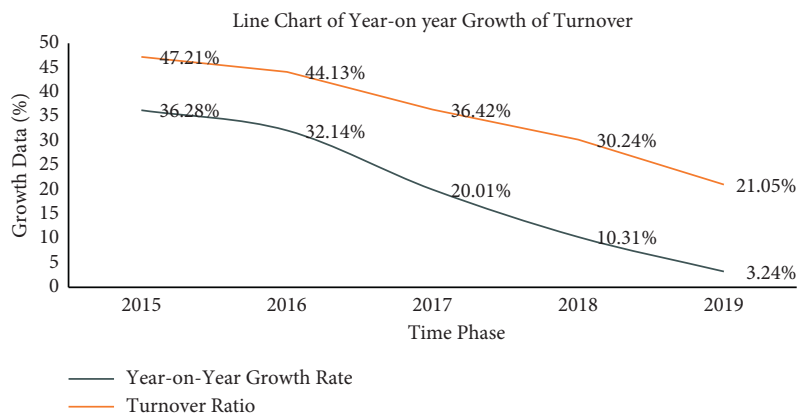

FIgURE 2: Line chart of year-on-year growth of turnover.

single marketing channel. Through the investigation, it is found that the current marketing of intelligent technology brands is still based on offline entity marketing, which is based on sales personnel and teams, which not only increases the marketing cost but also solidifies the marketing channel in face-to-face marketing, so that the marketing effect is greatly reduced. The second is the aging of traditional marketing methods. The abovementioned offline entity marketing greatly limits the scope of intelligent technology marketing, fails to provide a broader marketing space for products, and greatly restricts the sales of products. Second, the product type is single. The products of many smart technology brands are not only of few types but also similar to the products of other types of brands. The products produced are not featured or personalized and cannot meet the diversified demands of consumers for smart technology products, which restricts the marketing effect of smart technology products to a large extent. Third, the product price is on the high side. Intelligent technology products have higher requirements on production technology and higher costs of product development in the early stage. In addition, influenced by the traditional marketing mentioned above, their marketing costs are relatively high. All these lead to the high input cost of intelligent technology products and affect the final pricing of products. Generally speaking, the pricing of intelligent technology products is too high, which limits the consumer groups of such products and has a negative impact on the marketing effect.

\subsection{Marketing Strategy Optimization}

4.2.1. Fully Rely on the Internet. According to the above experimental results, we can find that the weak sales growth of intelligent technology products is the main problem faced by this type of brand at the present stage, and it is urgent to solve this problem. As the artificial intelligence is developed by relying on the Internet, the marketing of its intelligent technology products is also

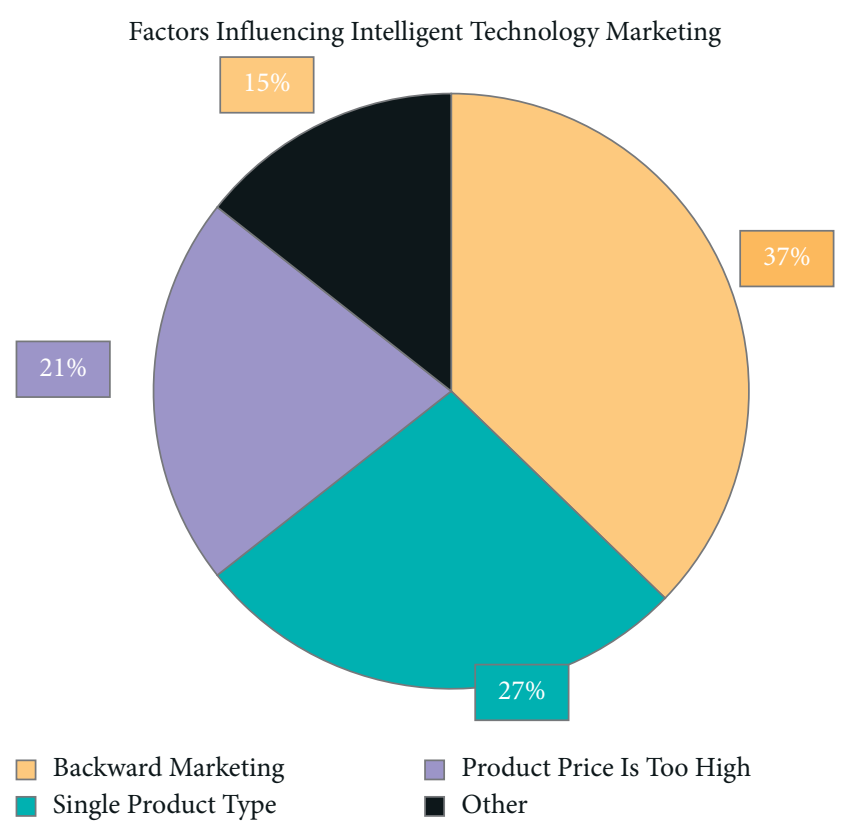

Figure 3: Analysis of factors influencing intelligent technology marketing.

inseparable from the Internet. To this end, intelligent technology companies should realize the continuous optimization of the traditional marketing model, relying on the Internet to develop a new marketing platform. In terms of marketing cost, accuracy, and authenticity, the mobile Internet of Things can effectively improve the marketing effect. Internet of Things marketing can be carried out from the following aspects: first, promote the innovation of traditional marketing channels. Marketing channel is an important part of the intangible assets of enterprises. In order to increase sales volume, it is necessary to promote the continuous updating of marketing channels. Under the background of the rapid development of the Internet, it is especially necessary to build the dual online and offline sales lines to realize the construction of three-dimensional sales network. Therefore, in addition to offline physical sales, smart technology brands should focus on the construction of a third sales platform, such as the establishment of a self-run online mall and the development of mobile clients. Compared with the traditional marketing channels, the new Internet channel has the characteristics of low cost and fast information capture. Second, promote the transformation of marketing methods. The marketing method directly affects the marketing effect. In the context of the Internet, intelligent technology companies can strengthen the use of event marketing with the help of modern communication means, attach importance to the enhancement of experiential marketing, strive to break through the limitations of traditional marketing methods, expand the potential user group of intelligent technology products, and promote the problem that the sales growth of such products is too slow. First of all, newspapers and other media can be used to recommend and publicize intelligent 
technology products. Second, promote the continuous strengthening of the relationship between each sales channel, so that it gradually turns to the partnership type of sales relations. Third, expand the scale of Internet marketing. Compared with the traditional marketing mode of intelligent technology, using the Internet to sell intelligent technology products is not only beneficial to colleges and universities and the realization of accurate marketing, but also can effectively reduce the labor cost of marketing. Various software applications are the main platforms and carriers of Internet marketing at the present stage, such as Microblog, WeChat, and other application software. Different marketing measures should be implemented according to different types of application software. These software platforms play a good role in promoting the marketing of intelligent technology products.

\subsubsection{Actively Implement the Product Development Strategy.} The development of intelligent technology has gone through many stages of change and is a gradual process. In recent years, with the improvement of people's living standard and the change of life style, their requirements on intelligent technology products are increasingly higher, and the speed of intelligent technology products upgrading is also faster and faster. Therefore, the development of intelligent technology products can only meet the needs of consumers to achieve rapid growth in sales. Thus, it can be seen that the development of intelligent technology products will directly affect the sales status of the products. First, the development of new products needs to follow a certain strategy. The first is the strategy of in-depth investigation. With the increasing number of intelligent technology companies and products, there are more and more homogeneous products. Once the homogenization of developed products occurs, it will directly affect the competitiveness of products and affect product sales. Therefore, prior to the development of new products, indepth market and consumer demand-oriented market research activities must be carried out. The second is the integrated packaging strategy. This strategy integrates product naming, packaging design, selling point determination, investment attraction, etc., which can promote the rapid promotion of product popularity and increase the speed of its entry into the market. Finally, there are strategies that echo national policies. The development of intelligent technological products should be consistent with the requirements of national policies. Second, the development of intelligent technology products needs to pay attention to the problems. On the one hand, it is necessary to promote the continuous improvement of the performance of intelligent technology products. The overall performance of the product has a direct impact on its market recognition and determines the final competitiveness of the product in the market. Therefore, in order to realize the promotion of product sales, it is necessary to start from the perspective of improving performance. The performance of intelligent technology products mainly includes compatibility, convenience, intelligence, and durability. On the other hand, promoting the continuous expansion of production lines is conducive to the sales of intelligent technology, so as to realize the personalized production of products and promote the continuous expansion of the $\mathrm{R} \& \mathrm{D}$ team.

\subsubsection{Reasonable Implementation of Pricing Strategy. The} price of products directly affects the sales of products, so the implementation of reasonable pricing strategy is conducive to the good marketing of intelligent technology products. The implementation of the pricing strategy mainly includes the following aspects: first, while ensuring the quality of the product, reduce the production cost as much as possible, so as to reduce the price level of the product and achieve the goal of "high quality and low price." Second, define the price of the product. There will be competition among similar intelligent technology products in the market. Therefore, the brand should accurately find the products with the same competitive advantages as the brand and determine the most competitive pricing by referring to the prices of other brands. Third, implement differentiated pricing. Unified brand will have different series of intelligent technology products because the production cost, market demand, purchasing ability, and other factors are different, especially the different market competitiveness, so there is a certain difference in price. Therefore, differentiated pricing should be adopted for different series of products in smart technology brands.

\subsubsection{Improve the Quality and After-Sales Service Quality.} At the present stage, because of the insufficiency of intelligent technology and the relatively low investment of some brands in research and development, many intelligent technology products are faced with certain quality problems. The existence of enterprises is closely related to the quality of products, and technology occupies the core position in the development of intelligent technology products. Therefore, to promote the continuous improvement of the sales status of intelligent technology products, it is necessary to fundamentally improve the quality of products. In addition, when consumers purchase goods, they pay special attention to the after-sales service of the product. The quality of the after-sales service of the brand directly affects the satisfaction degree of consumers and the return purchase rate.

With the help of big data analysis technology, the author predicts the marketing effect after the optimization of marketing strategy, compares the data before the optimization of marketing strategy, and finally obtains the data analysis results in Figure 4. Compared with before marketing strategy optimization, after optimization of intelligent technology products marketing strategy, the brand product sales rose year on year with a growth rate of around $7 \%$, the market share increased by about $26 \%$, and the overall marketing effect increased by $20 \%$. Thus, it can be seen that the marketing strategy optimization scheme proposed in this paper has certain feasibility and effectiveness. 


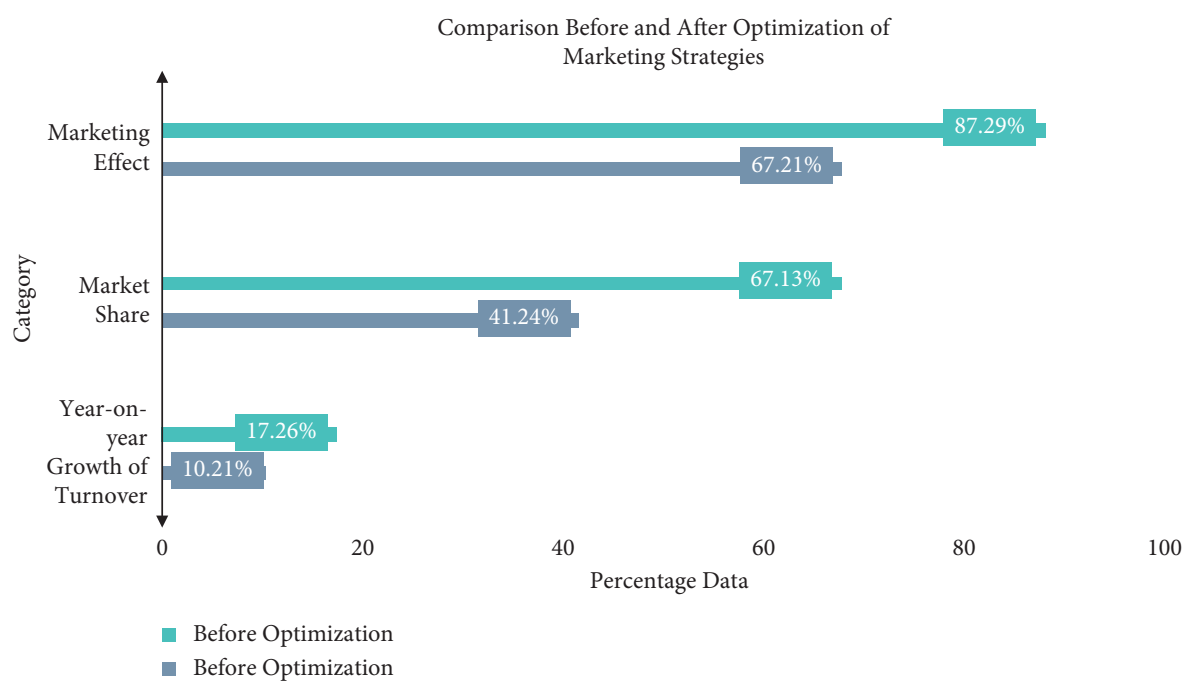

Figure 4: Comparison before and after optimization of marketing strategies.

\section{Conclusion}

With the continuous development of Internet technology and artificial intelligence, intelligent technology products have developed rapidly. However, influenced by many factors, the marketing status of such products in recent years is not ideal. Therefore, promoting the improvement of marketing strategy is an urgent problem to be solved in the development of intelligent technology products. Based on the analysis of the marketing status of intelligent technology brands under the background of artificial intelligence, this paper draws the following conclusions:

(1) In the recent years, the marketing effect of smart technology brands is poor, which is mainly affected by three factors: marketing method, product type, and product price

(2) This paper proposes specific strategies to optimize brand marketing of intelligent technology products from four perspectives, including Internet marketing, product quality, product pricing, and product development and tests the feasibility and effectiveness of this strategy with the help of big data analysis technology

\section{Data Availability}

The data that support the findings of this study are available from the corresponding author upon reasonable request.

\section{Conflicts of Interest}

The authors declare no potential conflicts of interest with respect to the research, authorship, and/or publication of this article.

\section{References}

[1] H. Parton, "Smart technology is transforming space," Property Week, vol. 86, no. 12, pp. 49-50, 2019.
[2] S. Moledina and A. Khoja, "Letter to the editor: digital dementia-is smart technology making us dumb?" The Ochsner Journal, vol. 18, no. 1, p. 12, 2018.

[3] A. Ho and O. Quick, "Leaving patients to their own devices? Smart technology, safety and therapeutic relationships," BMC Medical Ethics, vol. 19, no. 1, p. 18, 2018.

[4] N. L. Geller, D.-Y. Kim, and X. Tian, "Smart technology in lung disease clinical trials," Chest, vol. 149, no. 1, pp. 22-26, 2016.

[5] M. D. Seckeler, B. M. Gordon, and D. A. Williams, "Use of smart technology for remote consultation in the pediatric cardiac catheterization laboratory," Congenital Heart Disease, vol. 10, no. 6, pp. E288-E294, 2016.

[6] R. W. Treskes, L. A. Van Winden, N. Van Keulen et al., "Using smart technology to improve outcomes in myocardial infarction patients: rationale and design of a protocol for a randomized controlled trial, the box," Jmir Research Protocols, vol. 6, no. 9, p. e186, 2017.

[7] T. Cline and M. Marcusse, "Building a brand and telling a story in perpetual crisis: launching a sustainable archives marketing strategy," Public Services Quarterly, vol. 14, no. 3, pp. 265-274, 2018.

[8] P. B. Hirsch, "A breakfast of champions: brand marketing lessons from the great depression," Journal of Business Strategy, vol. 41, no. 4, pp. 63-67, 2020.

[9] J. Redler, "Building the counselling alliance," Renewing Pastoral Practice, vol. 19, no. 1, pp. 59-68, 2016.

[10] B. H. Wafler and Y. F. Badir, "Global products marketing strategy of two European MNCs in vietnam," The Journal of Product and Brand Management, vol. 26, no. 6, 2017.

[11] C. J. Yoo and J. Kim, "Case study: "bibigo" marketing strategy to be global no. $1 \mathrm{~K}$-food brand," Korea Business Review, vol. 20, no. 4, p. 183, 2016.

[12] M. Prado, "5 ways to boost your local online marketing strategy," Marketing Research, vol. 28, no. 1, pp. 14-15, 2016.

[13] A. Jeavons, "What is artificial intelligence?" Research World, vol. 2017, no. 65, p. 75, 2017.

[14] X. Yang, H. Li, L. Ni, and T. Li, "Application of artificial intelligence in precision marketing," Journal of Organizational and End User Computing, vol. 33, no. 4, pp. 209-219, 2021. 
[15] A. Bundy, "Preparing for the future of artificial intelligence," AI \& Society, vol. 32, no. 2, pp. 285-287, 2017.

[16] H. Lu, Y. Li, M. Chen, H. Kim, and S. Serikawa, "Brain intelligence: go beyond artificial intelligence," Mobile Networks and Applications, vol. 23, no. 2, pp. 368-375, 2017.

[17] M. Moravčík, M. Schmid, N. Burch et al., "DeepStack: expertlevel artificial intelligence in heads-up no-limit poker," Science, vol. 356, no. 6337, pp. 508-513, 2017.

[18] F. Jiang, Y. Jiang, H. Zhi et al., "Artificial intelligence in healthcare: past, present and future," Stroke and Vascular Neurology, vol. 2, no. 4, pp. 230-243, 2017.

[19] S. Makridakis, "The forthcoming artificial intelligence (AI) revolution: its impact on society and firms," Futures, vol. 90, pp. 46-60, 2017.

[20] L. Li and J. Zhang, "Research and analysis of an enterprise E-commerce marketing system under the big data environment," Journal of Organizational and End User Computing, vol. 336 pages, 2021.

[21] R. Li, Z. Zhao, X. Zhou et al., "Intelligent 5G: when cellular networks meet artificial intelligence," IEEE Wireless Communications, vol. 24, no. 5, pp. 175-183, 2017.

[22] T. G. Dietterich, "Steps toward robust artificial intelligence," AI Magazine, vol. 38, no. 3, pp. 3-24, 2017.

[23] R. Liu, B. Yang, E. Zio, and X. Chen, "Artificial intelligence for fault diagnosis of rotating machinery: a review," Mechanical Systems and Signal Processing, vol. 108, pp. 33-47, 2018.

[24] M. Hutson, "Artificial intelligence faces reproducibility crisis," Science, vol. 359, no. 6377, pp. 725-726, 2018.

[25] C. Cath, S. Wachter, B. Mittelstadt, M. Taddeo, and L. Floridi, "Artificial intelligence and the "good society": the US, EU, and UK approach," Science and Engineering Ethics, vol. 24, no. 7625, pp. 1-24, 2017.

[26] P. Glauner, J. A. Meira, P. Valtchev, R. State, and F. Bettinger, "The challenge of non-technical loss detection using artificial intelligence: a survey," International Journal of Computational Intelligence Systems, vol. 10, no. 1, pp. 760-775, 2017. 